\title{
Freud et l'approche psychanalytique : un autre regard sur la relation éducative
}

Freud and the psychoanalytic approach: Another look at the educational relationship

Freud y el método psicoanalítico : otra mirada sobre la relación educativa

\section{Mireille Cifali}

\section{(2) OpenEdition}

Édition électronique

URL : https://journals.openedition.org/ries/7141

DOI : 10.4000/ries.7141

ISSN : 2261-4265

Éditeur

France Education international

Édition imprimée

Date de publication : 1 décembre 2018

Pagination : 127-137

ISBN : $978-2-85420-620-3$

ISSN : $1254-4590$

\section{Référence électronique}

Mireille Cifali, «Freud et l'approche psychanalytique : un autre regard sur la relation éducative », Revue internationale d'éducation de Sèvres [En ligne], 79 | décembre 2018, mis en ligne le 01 décembre 2020, consulté le 24 juin 2021. URL : http://journals.openedition.org/ries/7141 ; DOI : https://doi.org/ $10.4000 /$ ries.7141 


\title{
Freud et l'approche psychanalytique : un autre regard sur la relation éducative
}

\author{
Mireille Cifali \\ Université de Genève
}

Peut-on considérer Sigmund Freud comme l'une des grandes figures de l'éducation dans le monde ? A-t-il influencé par sa pensée et sa conception du fonctionnement psychique la manière d'éduquer et d'enseigner? Nous pouvons répondre par l'affirmative. Peut-être pas tant lui que toutes celles et ceux qui se sont référés à son œuvre pour penser les modalités des métiers de la relation.

Penser l'éducation, ses déterminants et ses enjeux, en référence à la psychanalyse, relève d'un défi. Or ce défi a été relevé dès le début du vingtième siècle qui a vu naître la science du psychisme inconscient. Sigmund Freud lui-même a perçu la portée de ses découvertes pour les sciences humaines, y compris pour la pratique de l'éducation parentale et scolaire et, sur ses propres instigations, une éducation «subvertie » par la psychanalyse a été envisagée.

L'idée de Freud selon laquelle névroses et perversions fréquentes chez les adultes pouvaient être prévenues grâce à une éducation appropriée, non répressive des pulsions et faisant droit à la sexualité infantile jusque-là déniée, a suscité de grands espoirs dans le cercle des médecins qui fréquentaient l'illustre Viennois dès 1903. À la suite de Sandor Ferenczi, qui donne au Congrès des psychanalystes à Salzbourg, en 1908, une conférence intitulée "Psychanalyse et pédagogie ", d'autres membres du cercle viennois - Adler, Federn, Rank, Stekel, Tausk... - prennent conscience du "rôle énorme que joue l'éducation ", selon les propres mots de Freud.

En Suisse, et d'abord à Zurich où officiait Carl-Gustav Jung, les découvertes relatives au fonctionnement de l'inconscient suscitent très tôt de vifs débats. Des médecins, des pédagogues et des responsables de la formation des maîtres s'y réferrent. Parmi eux, le pasteur Oskar Pfister fut le défenseur d'une pédagogie " éclairée " par la nouvelle science, voire d'une application de la psychanalyse à l'éducation, dont celle-ci devait sortir transformée, grâce notamment à une meilleure connaissance des mécanismes inconscients qui gouvernent la psyché, celle de l'adulte éducateur comme celle de l'enfant à éduquer. À Genève, le fondateur de l'Institut Jean-Jacques Rousseau (1912), Edouard Claparède, fait rapidement une place à la psychanalyse dans l'enseignement de la science de l'éducation destiné aux enseignants. Y enseignent Charles Baudouin et Sabina Spielrein.

Les encouragements de Freud envers les efforts de diffusion d'une pédagogie dite psychanalytique ou à orientation psychanalytique, mise en œuvre dès après la Première Guerre mondiale par des personnalités telles l'instituteur bernois Hans Zulliger, les éducateurs viennois Siegfried Bernfeld et August Aichhorn, ne sont pas étrangers à la création, en 1926, d'une revue spécifique en langue allemande, la Zeitschrift 
für psychoanalytische Pädagogik. L'étude de cette riche publication - elle a cessé de paraître en 1937-a montré que s'y est inaugurée, autour de la parole et de l'inconscient qui la travaille, une collaboration originale entre des psychanalystes et des éducateurs.

Les années 1920-1930 sont très effervescentes sur le plan des rapports entre l'éducation et la psychanalyse et Freud s'en félicite. Il écrit dans la préface de Jeunesse à l'abandon de Aichhorn, en 1925 :

De toutes les utilisations de la psychanalyse, aucune n'a rencontré autant d'intérêt, éveillé autant d'espoirs et, partant, attiré autant de collaborateurs sérieux que son application à la théorie et à la pratique de l'éducation des enfants.

L’optimisme est de mise, en cette époque des commencements, et il est soutenu, dans les pays de langue allemande et en Suisse romande, par un intense mouvement de réforme pédagogique qui s’articule avec l'essor de la psychologie. Le courant dit de pédagogie psychanalytique s'inscrit de façon originale dans ce renouveau.

Les années trente, qu'inaugure la publication par Freud de Malaise dans la culture, marquent un retour au réalisme, voire à une forme de pessimisme. En cette époque d'illusion révolutionnaire et de montée des totalitarismes, Freud rappelle qu'il faut bien se familiariser « avec l'idée qu'il y a des difficultés inhérentes à l'essence de la culture et qui ne cèderont à aucune tentative de réforme ». Pourtant, il sait gré à sa fille Anna Freud de s'occuper de ce qu'il a lui-même négligé, à savoir « l'application de la psychanalyse à la pédagogie, à l'éducation de la génération suivante ».

Au fil du temps, la psychanalyse a ainsi transformé l'enseignement de même que l'enseignement dit spécialisé, elle a redessiné les prérogatives des métiers de la 128 relation et leur éthique; elle a influencé notre conception de l'enfance et le rapport des parents à l'éducation; elle a apporté une contribution particulière aux sciences humaines et aux sciences de l'éducation, à travers une démarche clinique. Sur plusieurs plans, un succès d'ensemble n'a manifestement pas été atteint, ce qui semble normal. Si la psychanalyse touche en effet à la construction de l'humain dans sa dimension psychique, on ne peut aboutir qu'à des succès locaux, avec tel ou tel enfant ou adolescent, avec telle ou telle classe, dans tel ou tel contexte. La construction psychique de chacun étant dépendante du contexte historique, des événements traversés, des idéologies, en bref d'influences diverses, elle ne cesse d'évoluer, dans sa norme et sa marge. Fabriquée par de multiples facteurs, elle se traduit, aux moments successifs de l'histoire, par des difficultés particulières d'exister et de se lier aux autres et au monde.

\section{DOMAINES D’INFLUENCES}

\section{L’autorité et la répression des pulsions}

Au début du XIX siècle, l'éducation est structurée par la soumission exigée, la répression des initiatives, l'intégration d'interdits brutaux, la formation d'un Surmoi créateur de conflits internes aboutissant à des névroses. La violence des gestes éducatifs est dénoncée par les psychanalystes, les coups sont bannis, l'autoritarisme du père se voit remis en question. Lever la chape de plomb qui pèse sur les enfants : tel est l'espoir d'une transformation des souffrances névrotiques dont les adultes énoncent la charge, 
lorsqu'ils parlent dans un cadre thérapeutique. Soumission ou liberté ? Obéissance ou libre choix ? Détermination ou laisser-faire ? Telle est l'alternative posée. Mais dès les années 1930, déjà, des critiques sont émises sur les effets d'une liberté sans limites. La psychanalyse n'est pas, à elle seule, à l'origine de la transformation du rapport à l'autorité, de sa fragilisation et de sa crise actuelle. Aujourd'hui, elle contribue à comprendre comment ne plus confondre autoritarisme, pouvoir et autorité par exemple ; à redessiner une obéissance qui ne s'oppose pas à la liberté ; et à revisiter la sanction comme prise de conscience des conséquences des actes posés et des douleurs possiblement causées (Bruno Robbes). C'est à elle qu'on s'adresse pour trouver la voie entre le Scylla et le Charybde de l'interdit et de la permission : le dialogue n'exclut pas la confrontation ; la " consistance » d'un adulte, capable d'un " non » structurant, permettrait de définir une autorité de « bonne veillance » (Daniel Marcelli).

\section{La sexualité et sa fantasmatique}

L'histoire de la psychanalyse est marquée par l'hypothèse freudienne d'une détermination sexuelle des névroses, entre fantasme et trauma. Une éducation sexuelle des enfants fut considérée comme capable de prévenir la fragilité psychique du rapport à l'amour sexué. Les psychanalystes se sont cependant rapidement aperçus qu'elle n'a pas le pouvoir, à elle seule, d'être préventive. Les informations données même au bon moment sont souvent des rationalisations refusées par un enfant qui persévère dans la croyance en ses propres théories fantasmatiques. Aujourd'hui, la plupart des enfants connaissent les techniques de l'amour sexué, ils font partie d'une société où les corps s'exhibent. Il semble, comme ils le disent parfois, "tout savoir ", quoique soumis à la normativité des images pornographiques et des impératifs de performance. Reste toujours en souffrance et en incertitude leur rapport à l'amour, à leur propre corps, à leur intimité, à la fragilité de leurs identifications, à l'altérité. Leurs questions portent invariablement sur les sentiments éprouvés, les peurs et les angoisses. L'information ne les protège guère des violences agies ou d'une soumission aliénante.

La psychanalyse a dénoncé les traumas - abus ou inceste - et les souffrances psychiques causées. Cette dénonciation a été relayée socialement : le secret peut se dévoiler mais aussi se manipuler pour fragiliser plus encore la relation de confiance qui devrait exister entre un adulte et un enfant. La méfiance est aujourd'hui de mise et cette vulnérabilité des adultes et de certains métiers a des conséquences néfastes sur leur possibilité d'action. Certes ces métiers comportent un risque de confusion de scènes et de sentiments. On le sait, même si on autorise rarement les professionnels à travailler un tel risque.

\section{Une transmission d'inconscient}

Un enfant hérite d'une part de l'inconscient parental. Il peut donc être tourmenté par ce qui ne lui appartient pas mais qui pourtant détermine sa vie. Forte de ce constat, la psychanalyse exhorte les parents à s'occuper de leur psychisme, espérant ainsi libérer leurs gestes éducatifs des projections et des répétitions, leur enjoignant de réaliser pour eux-mêmes une thérapie. L'hypothèse fonctionne, mais elle engendre une culpabilisation des parents, tenus pour responsables du devenir psychique de leurs 
enfants, avec la résistance que cette assertion reprise en accusation ne manque pas de provoquer. Une chose est donc d'énoncer que les adultes n'ont pas à mettre toute la faute sur le dos des enfants, une autre est de faire porter l'unique responsabilité sur les adultes. La place parentale est interrogée par la transformation de la famille, par la réduction des espaces publics, par l'avènement de professionnels qui savent mieux qu'eux leurs gestes, par le tissu social qui peine à ne pas les laisser à leur solitude. La psychanalyse tente d'en dessiner les repères possibles, sans catastrophisme ni alarmisme, en travaillant sur les processus d'idéalisation et le sens de la recherche de perfection.

Il faut aussi reconnaître que même des adultes analysés ne suffisent pas à faire grandir en toute sécurité, qu'il n'existe pas d'inconscient " épuré » qui soit sans effet, et que tout humain est contraint de traverser des épreuves psychiques comme la séparation, la mort, la disparition, l'amour, l'agressivité, etc., qui l'entraînent parfois dans la production de symptômes. En conséquence, chacun - parent comme professionnel - est dans la nécessité de mettre en travail et cet héritage inconscient et les conséquences des épreuves psychiques traversées, pour être plus à même d'accompagner celles et ceux qui viennent ensuite, en renonçant à croire que cela suffit à leur épargner de la souffrance.

\section{Pulsion de vie et pulsion de mort}

L'hypothèse freudienne d'une conflictualité psychique, d'un humain pris entre pulsion de vie et pulsion de mort, a été fortement débattue. Elle donne l'image pessimiste d'un humain qui ne se débarrassera pas de sitôt de ses capacités destructrices. Pourtant des générations de psychanalystes et de pédagogues ont rêvé de faire reculer les actes barbares, et même jusqu'aux guerres. Ils ont travaillé l'agressivité nécessaire à vivre et la fabrication de la violence. S’ils ont été déçus dans leur espoir de transformer la destructivité à l'œuvre dans les affaires humaines, ils ont compris par là même que cette tension entre construction et destruction est condamnée à être accompagnée pour que la haine ne s'enkyste pas, que l'indifférence objectivante ne se transforme en anéantissement d'un autre vivant, et que l'agressivité ne fasse pas peur mais trouve son cadre et ses règles pour être mise au service des pulsions de vie et non de mort.

L'ambivalence des sentiments ne cesse d'être rejetée, par les parents comme par les jeunes professionnels. On croit à l'amour et on ne tient pas la haine comme constitutive de l'humain. La psychanalyse travaille ce déni, pour empêcher que le refoulé ne provoque l'inacceptable toujours surgissant dans le rapport à un autre.

\section{Le processus d'apprendre}

La psychanalyse a fait l'hypothèse d'une absence de coupure entre l'affect et le cognitif, entre les sentiments et le rationnel, l'une pouvant dynamiser l'autre ou, au contraire, l'inhiber. Le symptôme scolaire n'est dès lors pas seulement lié aux connaissances à acquérir mais aussi à la position affective dans le savoir, au rapport au savoir, à la confusion imaginaire des scènes, aux dynamiques transférentielles. Des psychanalystes soulignent alors les conséquences de cette intelligence troublée : peur d'apprendre ; angoisse face à ce qui ne se sait pas encore ; investissements fantasmatiques des matières scolaires ; interdit de savoir. L'élève est un sujet apprenant, il peut être un enfant en difficulté et un élève en souffrance. La notion de cancre est mise en 
doute, et le passage au-delà de la difficulté scolaire n'est pas seulement rendu possible par des leçons de rattrapage mais aussi par l'accompagnement d'un thérapeute.

Cette hypothèse a-t-elle eu des répercussions sur le métier d'enseigner ? Certainement, pour qui s'intéresse aux processus psychiques liés à l'apprendre. Mais elle contribue, malgré elle, à véhiculer la croyance en une causalité simple qui semble arranger parfois les professionnels de l'enseignement. Si un élève est en difficulté, alors la cause vient de l'affectif et donc de sa famille responsable de son état psychique ; il s'agit de l'envoyer chez un thérapeute avant qu'un enseignant ne reprenne sa tâche. Au lieu de rendre attentif un enseignant à trouver les dispositifs aidant celui qui se trouve en difficulté, on aboutit parfois à une impasse et à l'abandon de tout travail avec cet élève dans l'espace d'une classe. Cette réaction n'est pas générale, elle est combattue par celles et ceux qui cherchent à comprendre la complexité de l'instant d'apprendre, ces professionnels étant souvent à la marge de l'institution scolaire.

L'autre reproche, formulé par ceux qui pensent que la transmission du savoir doit se délester de toute interprétation " psychologisante », est d'avoir fait perdre à la fonction d'enseignement la crédibilité qu'elle tire du savoir et de sa transmission. Un tel procès n'aide aucunement à envisager les composantes actuelles de la difficulté à enseigner et à apprendre. L'importance reconnue de la composante affective de l'apprentissage et de l'engagement subjectif d'un élève dans son rapport au savoir est en effet porteuse d'une autre manière d'enseigner et d'être intéressé par la difficulté éprouvée non seulement en termes d'évaluation et de relégation mais aussi comme production nécessaire d'un élève à un certain moment de son parcours. Si on convient que ni la technique ni le savoir ne sont à négliger, l'intérêt se porte également sur ce qui pousse à savoir ou à refuser de savoir ; ce qui fait joie de penser et jubilation de chercher et de comprendre ; sur les chemins de traverse que prennent l'investissement et le désinvestissement du savoir.

\section{INFLUENCES DANS LES SCIENCES DE L’ÉDUCATION}

\section{Une posture clinique}

Une des influences majeures de la psychanalyse dans les sciences de l'éducation, comme dans d'autres domaines, se réalise à travers une " posture clinique ». Une démarche clinique n'appartient pas à une seule discipline, elle ne constitue pas un terrain spécifique. C'est un art de la recherche, de l'intervention et de la formation visant un changement et se tenant dans la singularité des situations rencontrées. Elle concerne donc tout autant le professionnel qui tente de penser son action que le chercheur accompagnant le professionnel dans son désir de comprendre et l'intervenant cherchant à faire évoluer une situation, une structure, une institution.

Quand on se confronte à des situations où le but premier n'est pas de construire des connaissances généralisables mais où l'enjeu est de permettre que l'autre guérisse, accède au savoir, dépasse une difficulté handicapante, se dés-asujettisse ; où s'élabore ainsi, avec les interlocuteurs en présence, une compréhension impliquée de ce qui se passe, une co-construction d'un sens qui provoque du changement, alors on se trouve 
dans un espace qualifié de " clinique ». Il s'agit là de postulats épistémologiques qui autorisent, entre autres, le travail de la subjectivité et la construction de connaissances dans l'action. Cette posture dans le domaine du soin, de l'enseignement et de l'éducation entre de fait en tension avec l'habitus scientifique, elle préserve en tous les cas un espace de débats bénéfiques.

\section{Une subjectivité assumée, non sans une intersubjectivité}

Cette manière particulière de construire du savoir à partir des gestes quotidiens posés avec d'autres invite un professionnel à écouter ce qui travaille sans qu'il le sache, à ne pas s'en tenir à une unique rationalité, à accepter les tensions entre des contraires et à lui faire reconnaître l'impact de sa subjectivité dans l'action. Cette subjectivité se travaille sans qu'elle se confonde avec l'ego ou l'identité. On pourrait effectivement soupçonner la psychanalyse de renforcer ici le mouvement d'une individualisation qui rabat le sujet sur sa psychologie, l'instituant centre du monde et fragilisant d'autant la norme et le lien social. Reconnaître et travailler la subjectivité, c'est au contraire partir d'un «moi » pour ne pas aboutir à « moi ».

Cette subjectivité assumée et travaillée concerne autant le professionnel dans l'action que celui qui construit avec lui une compréhension de ce qui se passe. Elle fonde une position qui cherche à aboutir à de l'objectivité par la confrontation des subjectivités et non pas leur éviction. Elle signe cette posture clinique que la psychanalyse a renouvelée. La science est en effet portée à refouler la vie intérieure et inconsciente des humains. L'une des fonctions de la psychanalyse est d'empêcher qu'un tel refoulement ne réussisse tout à fait, aujourd'hui comme demain.

Si l'on retient une "subjectivité » travaillée, s'ensuit un soin pris également envers une intersubjectivité, c'est-à-dire une prise en compte de la dimension relationnelle des métiers, avec reconnue l'importance d'une "rencontre » qui pousse hors de soi. La relation entre les humains a été jusqu'ici partie prenante de la guérison, du possible passage de la difficulté présente. Nous aurons beau avoir des pilules pour chaque dysfonctionnement repéré socialement, l'humain éprouve la nécessité de construire du sens à ce qui lui arrive et de s'arrimer à quelqu'un pour se risquer à exister. Prendre soin de la relation, c'est aussi tenter de comprendre les forces qui l'animent comme pouvant être destructrices. Le rapport à un autre (en singulier ou au pluriel) est souvent douloureux, le travailler préserve et soi et un autre dans un contexte institutionnel.

\section{Une éthique}

La psychanalyse a développé, dans le champ de l'éducation, une éthique de l'altérité et de la singularité ; elle a engagé une réflexion constante sur la «bonne » distance avec un autre; elle a exigé que l'implication subjective ne soit pas tue mais élaborée ; elle a requis un travail sur soi pour rencontrer professionnellement l'autre, renvoyé chacun à ses projections et nommé les effets d'un transfert et contre-transfert ; elle a questionné la dépendance et le pouvoir, tracé le bénéfice des limites, désigné les effets de l'idéal, traqué la vérité et ses retombées. Par sa clinique, elle dégage nombre de professionnels d'une dichotomie entre théorie et pratique, dessine 
une théorie qui n'a pas à s'appliquer et un vivant qui toujours l'excède ; elle développe une construction de connaissances à même l'action.

Poser son apport en termes d'éthique revient à considérer cette approche sous l'angle d'un questionnement, d'une ouverture pour ceux qui se confrontent à l'incertitude et ne la supportent pas toujours. On déconstruit pour laisser respirer ; on nomme les vérités présentes en traçant les filiations au travers des siècles ; on relativise en ne cessant pas de croire à une passion du savoir ; et ainsi s'instaure une constante humilité dans l'usage du savoir accumulé.

\section{Une articulation entre psychique et social}

La psychanalyse est souvent entrevue comme centrée sur l'intériorité, le psychisme individuel, et comme se désintéressant de la question sociale. Liée à la relation duelle, elle ne saurait ainsi rien apporter ni au groupe, ni à la société avec ses institutions et ses organisations.

Si cette critique est apparue très tôt, dénonçant un manque des psychanalystes dans la prise en compte du groupe et de ses effets, aujourd'hui de nombreux travaux réalisés soulignent que la construction du sujet advient dans l'intersubjectivité ; que l'intérieur et l'extérieur ne peuvent être séparés si on veut comprendre leur construction; que l'institution détermine un sujet alors contraint à un travail de lucidité pour se désaliéner.

Certains de ces travaux s'attachent alors à mettre en perspective les dimensions imaginaires agissant entre un sujet et une institution. Le groupe, le cadre, les médiations sont reconnus comme étant des structurants essentiels, à l'origine du dépassement des difficultés, que ce soit au sein d'une classe, d'une école, d'une équipe ou d'une institution.

\section{Des dispositifs de formation}

En accord avec les différentes lignes de tension ci-dessus nommées, se sont construits des dispositifs originaux de formation pour penser l'action pédagogique dans toutes ses dimensions.

Ce qui se nomme aujourd'hui " analyse de pratiques » doit probablement davantage à la psychanalyse qu'il n'y paraît. Partir des situations du vivant pour construire de la connaissance est un mouvement travaillé et pensé dans le cadre d'une posture clinique. Celui qui accompagne une analyse des pratiques professionnelles quitte certaines de ses positions de formateur : comme celle de transmettre un savoir $a$ priori, pour faire avec ce qui vient, dans la surprise et l'inattendu, en cherchant à relier ce qui est disjoint et en honorant les détails et le banal. Il s'agit d'accompagner un professionnel dans le travail sur la singularité d'une situation où il est engagé, parfois même aveuglé, ce qui lui rend possible de comprendre après coup ce qui s'est passé et de se déplacer. Un formateur est alors garant de la construction d'une éthique, d'une manière de se guider là où la solution n'est pas tout de suite apparente.

À travers des récits, des monographies où s'interroge l'opposition de la science et de la littérature, une attention est portée à l'écriture de cette compréhension construite à partir de l'expérience. 


\section{Des PERSONNeS, DES MOUVEMENTS ET DES LIEUX ${ }^{1}$}

\section{Une présence dans des pays et des institutions}

La présence d'un apport de la psychanalyse à l'éducation et l'enseignement est le plus souvent corrélée à l'influence exercée par la psychanalyse dans un pays.

$\mathrm{Si}$, en son commencement, sont concernés des pays comme l'Allemagne, l'Angleterre, l'Autriche, la France, la Russie, la Suisse ; d'autres pays européens s'y sont adjoints, comme la Belgique, l'Espagne, le Portugal, la Grèce, l'Italie ; en Amérique du Sud, des pays sont concernés, comme l'Argentine, le Brésil (avec la Revista Estilos da clinica), le Mexique, l'Uruguay; en Amérique du Nord, les États-Unis et le Canada ${ }^{2}$. Son absence dans les pays arabo-musulmans et des pays ex-communistes est évidemment due à l'interdiction de la psychanalyse considérée comme science subversive.

Dans chaque pays opère à chaque fois une filiation à partir de personnes ayant fait une thérapie psychanalytique et ancrées, parfois préalablement, dans le monde de l'enseignement ou de l'éducation. Inscrites dans des institutions, universitaires ou non, elles font alors transmission et impriment une orientation particulière à la conception de cette approche. Il s'agit donc d'un apport pluriel des découvertes freudiennes et non d'un mouvement d'ensemble. Chacun des auteurs cités dans cet article, et d'autres encore, ont eu et ont encore une influence sur la conception des « métiers de l'humain », afin qu'ils puissent trouver à penser leurs gestes professionnels en travaillant leurs dimensions relationnelles et affectives.

Parmi les principales institutions concernées, nous relevons :

- les lieux de formation des enseignants, des éducateurs, des éducateurs spécialisés et des rééducateurs, des éducateurs du jeune enfant, des travailleurs sociaux, des psychologues cliniciens, des orthophonistes, des infirmiers en santé mentale ;

- les services médicaux pédagogiques dans l'accueil des enfants en difficultés scolaires ;

- les institutions supérieures d'enseignement.

Dans les sciences de l'éducation et de la formation, cette approche est portée par :

- des équipes de recherche et d'enseignement en sciences de l'éducation qui intègrent dans leur intitulé la référence à la psychanalyse ou à une démarche clinique ;

- la présence, depuis 2003, dans le Réseau international de recherche en éducation et en formation (REF) d'un symposium clinique dont sont issus des ouvrages collectifs ;

- par des orientations cliniques dans la didactique des mathématiques, la didactique du sport, la didactique de l'histoire, la recherche biographique, les récits d'expérience, l'intervention institutionnelle, et plus spécifiquement la relation pédagogique avec sa part affective (émotion, sentiment), etc.

1. Les références mentionnées dans cette partie et en annexe 1 ne sont pas exhaustives.

2. Voir l'annexe 1. 


\section{Une présence à travers des mouvements et des revues}

En filiation directe de Sigmund Freud, il s'agirait d'interroger l'influence qu'ont exercée Anna Freud et Mélanie Klein en Angleterre et ailleurs. Nous ne le ferons pas. Il convient aussi de souligner celle très importante de Donald Woods Winnicott et de Wilfred Bion. Nous nous contenterons ici de relever les mouvements qui, depuis la France, ont disséminé dans d'autres pays.

En France, cet apport a été rendu possible grâce à la diffusion de la psychanalyse dans un certain milieu intellectuel par l'intermédiaire de Jacques Lacan, de ses séminaires et de ses écrits. En théorisant longuement sur l'assomption de la parole et les ruses de l'inconscient, il a renouvelé la lecture de Freud et influencé de nombreux disciples. En ce qui concerne le lien entre éducation et psychanalyse, c'est à deux de ses élèves, Maud Mannoni et Françoise Dolto, que revient le mérite d'avoir considérablement contribué à modifier le regard et les attitudes des parents et des éducateurs envers les enfants et les adolescents en souffrance.

Dans les courants pédagogiques, c'est la pédagogie institutionnelle (PI) qui a explicitement fait de l'inconscient l'un de ses piliers. Élaborée à son commencement par Fernand Oury et Aïda Vasquez, elle a été sensible à la dimension thérapeutique de l'espace institutionnel, en lien également avec le travail mené par Jean Oury à la Clinique de La Borde. La PI est représentée par plusieurs courants ${ }^{3}$ et on la retrouve dans de nombreux pays : Allemagne, Brésil, Belgique, Canada, France, Italie, Japon, Luxembourg, Grèce Suisse. Une place particulière concernant la référence à Freud revient à Francis Imbert, professeur de philosophie et psychanalyste à l'institut universitaire de formation des maîtres (IUFM) de Créteil, qui a développé une remarquable pensée sur la praxis pédagogique et la question de l'éthique dans le champ éducatif et qui, avec le Groupe de recherche en pédagogie institutionnelle (GRPI), a publié de nombreuses monographies sur leur pratique dans la classe. Nous pouvons également évoquer l'expérience précieuse de l'École de la Neuville en France (Françoise D’Ortoli et Michel Amram), supervisée à ses débuts par Françoise Dolto. Remarquons encore que la pédagogie Freinet n'a pas été sans être influencée par les découvertes psychanalytiques (Jovenet), même si certains s'en défendent.

Autour de la revue française Cliopsy $y^{4}$, en filiation avec Claudine BlanchardLaville, didacticienne des mathématiques, se sont fédérées de nombreuses recherches cliniques d'orientation psychanalytique en sciences de l'éducation, que ce soit en France, Angleterre, Argentine, Brésil, Espagne, avec un fort ancrage universitaire en France.

Les groupes dits de "Soutien au Soutien » créés par le psychologue et psychanalyste Jacques Lévine au début des années 1970 et constitués en association (AGSAS) en 1993, proposent sur tout le territoire français, ainsi qu'au Portugal et au Liechtenstein, des groupes qui cherchent à démêler la complexité de ce qui se passe au niveau relationnel où ils sont subjectivement impliqués. Jeanne Moll en a été longtemps la présidente. Leur revue s'intitule Je est un autre ${ }^{5}$.

3. Voir : https://reseau-pi-international.org/

4. Revue Cliopsy : https://www.revuecliopsy.fr/

5. Voir : http://agsas-ad.fr/. 


\section{Une persistance}

Faut-il concevoir cette approche comme étant dépassée, même caduque, comme le serait la psychanalyse ? Remplacée par les conceptions actuelles de l'apprentissage par exemple, en particulier par la neuropsychologie ? Certains le pensent, d'autres œuvrent pour préserver une articulation des deux approches comme nous le démontre Michelle Bourassa. Notre conviction reste que comprendre et travailler la dimension relationnelle et affective demeurera toujours nécessaire. On peut douter qu'une approche neuropsychologique des apprentissages et des souffrances psychiques réduise définitivement leur part énigmatique.

L'expérience des liens entre psychanalyse et éducation a donné lieu à bien des connaissances sur les dérives possibles, sur les prudences à conserver, ces connaissances concernent toute approche théorique qui veut, à elle seule, transformer les pratiques enseignantes. Nous aurions intérêt à nous en souvenir, pour ne pas répéter ce que l'on sait être des erreurs peu bénéfiques.

Nous avons surtout la nécessité renouvelée de préserver les processus de création et d'humanisation. La transmission d'humanité n'est pas automatique, l'éthique psychanalytique en est l'une des garantes, avec la philosophie.

\section{RÉFÉRENCES BIBLIOGRAPHIQUES}

BLANCHARD-LAVILlE C., CHAUSSECOURTE Ph., HATCHUEL F., PECHBERTY B. (2005) : «Recherches cliniques d'orientation psychanalytique dans le champ de l'éducation et de la formation ", Revue française de pédagogie, $\mathrm{n}^{\circ} 151$.

BRIZMAND. (2010) : Freud and Education, Abingdon, Taylor \& Francis Ldt.

CIFALI M. (1982) : Freud pédagogue? Psychanalyse et éducation, Paris, InterEditions.

FILLOUX J.-C. (2000) : Champ pédagogique et psychanalyse, Paris, Puf.

IMBERT F., Cifali M. (1998) : Freud et la pédagogie, Paris, Puf.

MANZ B. (2013) : "Geschichtlicher Abriss sur psychoanalytischen Pädagogik », dans F. Imbert et M. Cifali, Freud und die Pädagogik, Mit Texten von Sigmund Freud, August Aichhorn und Hans Zulliger, Frankfurt Brandes et Apsel.

MILHAUD-CAPPE D. (2007) : Freud et le Mouvement de Pédagogie psychanalytique, Paris, Vrin.

MOLL J., CIFALI M. (2003) : Pédagogie et psychanalyse, Paris, Dunod, 1985, rééd. Paris, L'Harmattan.

MOLL J. (1989) : La pédagogie psychanalytique. Origine et histoire, Paris, Dunod.

PAIN J. (2017) : « 1967-2017. Vers une pédagogie institutionnelle. Cinquante ans après. Quelle actualité des approches institutionnelles aujourd'hui ?», en ligne [https://bit.ly/2AugutU]. 


\section{Annexe 1. Une présence dans des pays et des institutions (sans exhaustivité)}

\section{Allemagne}

La Zeitschrift für psychoanalytische Pädagogik; puis, à partir d'August Aichhorn, s'est instaurée une filiation pour l'accueil des enfants et adolescents en difficultés sociales, la Sozialpädagogik; l'Association allemande des sciences de l'éducation (DGfE) a, depuis 1993, une commission pour la pédagogie psychanalytique (Kommission psychoanalytische Pädagogik). Citons Burkhard Müller (Université Hildesheim); Stephan Becker (Université de Tübingen); Hilde Kipp (Université de Kassel). Angleterre

L'Institut Anna Freud National Centre for Children and Families ; Mélanie Klein Trust ; l'École Summerhill d'Alexandre Neill (Londres).

\section{Argentine}

Marta Souto (Université de Buenos-Aires).

\section{Autriche}

Le Centre Rudolf Ekstein, à Vienne.

\section{Belgique}

Guy de Villers (Université de Louvain-la-Neuve).

Brésil

Leandro de La Jonquière (Université de Saô Paulo, Paris 8), co-fondateur de la Revista Estilos da clinica.

\section{Canada}

Michelle Bourassa (Université d’Ottawa) ; Deborah Brizman (Université York, Toronto).

\section{Espagne}

José Luis Atienza (Université d'Oviedo).

\section{États-Unis}

De nombreux psychanalystes allemands et autrichien juifs ont émigré aux États-Unis, comme Fritz Redl (qui a fondé à Détroit la Pioneer House); Bruno Bettelheim (École orthogénique de l'Université de Chicago) ; Erik H. Erikson ; Siegfried Bernfeld (pionnier de l'Éducation nouvelle); Rudolf Ekstein.

\section{France}

Jacky Beillerot, Claudine Blanchard-Laville, Françoise Bréant, Louis-Marie Brossard, Jean-Claude Filloux, Catherine-Françoise Hatchuel, Nicole Moscovici, Jacques Natanson, Catherine Yelnik (Université Paris-Nanterre) ; Philippe Chaussecourt, Janine Filloux, Bernard Pechberty (Université Paris-Descartes) : Florence Gararini, Patrick Geffard, Ilaria Pirone Université Paris 8 Vincennes-Saint-Denis); Florence Giust-Despraires (Universités de Paris 8 et Paris-Diderot) ; Arnaud Dubois (Université de Clergy-Pontoise) ; Sébastien Ponnou (Université de Rouen) ; Jacques Terrisse (Université Toulouse Jean Jaurès) ; Christophe Niewiadomki (Université de Lille) ; Denis Loizon (Université de Bourgogne) ; Francis Imbert (IUFM de Créteil) ; Marie-Claude Baïetto (IUFM de Grenoble) ; Yves de la Monneraye (IUFM de Nantes) ; Carmen Strauss-Raffy (IUFM d'Alsace) ; Jeanne Moll (IUFM de Strasbourg) ; Joseph Rouzel, Jeanne Duval Héraudet (Institut européen psychanalyse et travail social).

\section{Liechtenstein}

Beat Manz.

\section{Portugal}

Maria Teresa Casanova de Araujo Sà (Escola Superior de Educação de Santarèm).

\section{Russie}

Vera Schmidt, L'éducation psychanalytique en Russie soviétique, rapport sur le jardin d'enfants expérimental de Moscou, créé en 1921 et auquel a également participé Sabina Spielrein.

\section{Suisse}

Oscar Pfister ; Hanz Zulliger ; Charles Baudouin ; Ernst Schneider (École normale de Berne) ; Mireille Cifali (Université de Genève) ; Reinhard Fatke (Université de Zürich); Jean-Marie Cassagne, Bessa Myftiu (HEP Lausanne). 
